\title{
Interactions entre bactéries lactiques mésophiles dans le lait : rôle des facteurs nutritionnels
}

\author{
Vincent Juillard, Catherine Foucaud, Bénédicte Flambard, \\ Sylviane Furlan, Pascale Bellengier, Jean Richard
}

Unité de recherches laitières, Inra, 78352 Jouy-en-Josas cedex, France

\begin{abstract}
Incidence of nutritional factors on the interaction between mesophilic lactic acid bacteria during growth in milk. The importance the nutritional factors have on the interactions between mesophilic lactic acid bacteria during associative growth in milk is demonstrated by analyzing three examples of mixed cultures used in cheese technology. (i) The interaction between proteinase-positive and proteinase-negative strains of Lactococcus lactis results from a competition for the utilization of casein-derived peptides. The extent of the competition depends on the balance between the two types of cells, the proteolytic activity of the proteinase-positive strain, and the type of proteinase the strain produces. (ii) The interaction between proteinase-positive strains of $L$. lactis is influenced by the type of proteinase $\left(\mathrm{P}_{\mathrm{I}}\right.$ - or $\mathrm{P}_{\mathrm{m}}-$ type $)$ the strains produce. A competition for the use of casein-derived peptides, and a reduction in proteolytic activity of the $P_{1}$-type proteinase strain were presumably responsible for the interaction. (iii) Finally, the effects of co-culturing lactococci and leuconostocs are apparently strain-dependent. Nevertheless, a competition between both species for peptide utilization seems to be a frequent feature of such mixed cultures. These three examples emphasize the major role of the proteolytic activity of L. lactis when co-cultured in milk. (C) Inra/Elsevier, Paris.
\end{abstract}

\section{lactococci / proteinase / leuconostoc/ milk / interaction}

Résumé - Trois exemples, inspirés des problèmes rencontrés par la technologie fromagère, illustrent l'importance des facteurs nutritionnels dans les interactions entre bactéries lactiques mésophiles constitutives d'un levain. L'interaction entre souches protéolytiques et variants non protéolytiques de lactocoques résulte d'une compétition pour les produits de dégradation des caséines, compétition dont l'intensité dépend de la proportion relative des deux types de cellules, du niveau d'activité protéolytique de la souche protéase-positive et du type de protéase qu'elle synthétise. L'interaction qui se produit entre souches protéolytiques de lactocoques est également régie par le type de protéase de paroi synthétisée. Elle semble résulter d'une compétition pour l'utilisation des produits de protéolyse, au détriment de la souche ayant une protéase de type $\mathrm{P}_{1}$, et d'une inhibition de la synthèse de cette protéase par les peptides accumulés par l'autre type de protéase pendant la culture. Enfin, contrairement aux deux exemples précédents, les interactions entre lactocoques et leuconostocs semblent dépendre des souches en présence. Néanmoins, l'existence d'une compétition entre les deux espèces pour l'utilisation des nutriments azotés est fréquente. L'ensemble de ces résultats souligne l'importance de l'activité protéolytique des lactocoques dans les phénomènes d'interactions lorsque ces microorganismes sont cultivés en association dans du lait. (C) Inra/Elsevier, Paris.

lactocoque / protéase / leuconostoc / lait / interaction 


\section{INTRODUCTION}

Les interactions entre microorganismes évoluent en fonction des souches qui sont associées. De plus, elles sont étroitement dépendantes des conditions de l'environnement, en particulier du milieu et des conditions de culture $[2,7]$. Cela se comprend aisément, puisque les principales causes d'interactions entre microorganismes dérivent de leurs propriétés physiologiques, dont l'expression est étroitement dépendante des conditions de culture. Les bactéries lactiques sont des microorganismes exigeants d'un point de vue nutritionnel. Parmi les facteurs responsables d'interactions entre souches, les facteurs nutritionnels prennent donc une place importante [9]. Plus précisément, la croissance des bactéries lactiques dans le lait est principalement régie par leur aptitude à s'approvisionner en acides aminés -[10]. Comme l'illustrent les trois exemples inspirés par la technologie fromagère discutés ci-après, la nutrition azotée des bactéries lactiques en général, et des lactocoques en particulier, est à l'origine de divers phénomènes d'interaction, dont la nature et les effets varient selon les associations considérées.

\section{INTERACTION ENTRE SOUCHES PROTÉOLYTIQUES ET VARIANTS NON PROTÉOLYTIQUES DE LACTOCOCCUS LACTIS}

La présence de variants Prt au sein d'une culture de souches $\mathrm{Prt}^{+}$est un phénomène connu depuis longtemps [5]. L'interaction qui se produit entre ces deux types de cellules présente une caractéristique générale, à savoir la stimulation du variant Prt $[11,15]$. Le variant Prt étant incapable d'utiliser les caséines du lait comme source d'acides aminés, son développement en culture pure ne repose donc que sur l'utilisation des substrats azotés non protéiques du lait. Si des cellules $\mathrm{Prt}^{+}$ sont présentes dans la culture, elles dégradent les caséines. Les peptides, libérés dans le milieu de culture, peuvent donc être utilisés par les deux types de cellules en présence. Naturellement, plus la proportion de cellules $\mathrm{Prt}^{+}$dans le mélange est importante, plus la stimulation du variant Prt est marquée [8]. Cette relation aurait pu être qualifiée de commensalisme au sens défini par Meers [12] ou Fredrickson [4], si le développement de la souche $\mathrm{Prt}^{+} \mathrm{n}^{\dagger}$ était pas lui-même affecté. Comme la vitesse de croissance de la souche $\mathrm{Prt}^{+}$en culture pure est limitée par la vitesse de dégradation des caséines par la protéase de paroi [6], la quantité de substrats disponibles pour les cellules $\mathrm{Prt}^{+}$ durant la coculture est amputée de la part consommée par les cellules Prt. Il en résulte que la vitesse de croissance de la souche $\mathrm{Prt}^{+}$est ralentie. L'intensité de cette compétition est d'autant plus marquée que la proportion de cellules compétitrices (souches Prt) dans le mélange est importante [8].

L'interaction entre souches $\mathrm{Prt}^{+}$et Prt peut donc être définie comme un « commensalisme compétitif », dont l'intensité dépend de la proportion relative des deux souches dans le mélange. Néanmoins, d'autres facteurs influent sur l'intensité et la nature de l'interaction, parmi lesquels le niveau d'activité protéolytique exprimé par les souches $\mathrm{Prt}^{+}$[11]. En effet, plus la protéase de paroi libère de peptides, plus la stimulation du variant Prt sera marquée et/ou moins l'inhibition de la souche $\mathrm{Prt}^{+}$ sera sensible. Pour des raisons non encore élucidées, les caractéristiques de l'interaction entre souches $\mathrm{Prt}^{+}$et variants Prt dépendent également du type de protéase synthétisée par la souche $\mathrm{Prt}^{+}[11]$. En effet, à niveau d'activité protéolytique égal, l'intensité de la compétition (estimée par le degré de réduction du taux de croissance de la souche $\mathrm{Pr}^{+}$) est plus forte lorsque la souche $\mathrm{Prt}^{+}$synthétise une pro- 
téase de type $\mathrm{P}_{\mathrm{I}}$ que si la protéase est de type $\mathrm{P}_{\mathrm{III}}$ (figure 1). Aucune corrélation n'ayant pu être mise en évidence entre l'intensité de la stimulation des souches Prt $^{-}$et le type de protéase de la souche associée, cela suppose que l'effet commensal, contrairement à l'effet compétitif, ne dépend pas du type de protéase. L'explication de ce phénomène requiert une meilleure connaissance de la nature des peptides libérés à partir des caséines par une protéase de type $\mathrm{P}_{\mathrm{III}}$.

\section{INTERACTION ENTRE SOUCHES PROTÉOLYTIQUES DE LACTOCOCCUS LACTIS}

L'influence du type de protéase de paroi sur le déroulement de cultures binaires associant deux souches $\mathrm{Prt}^{+}$de L. lactis a également été mis en évidence [3]. Deux souches génétiquement modifiées, ne différant que par le type de protéase synthétisée, ont été utilisées comme modèle d'étude. Leur culture en mélange aboutit à un déséquilibre systématique de la population en faveur de la souche produisant une protéase de type $\mathrm{P}_{\mathrm{III}}$ (figure 2 ). Des résultats similaires étant obtenus lors de la coculture de souches naturelles, ce déséquilibre semble donc caractériser l'interaction entre souches $\mathrm{Prt}^{+}$produisant des protéases de types différents. Il résulte d'une diminution du taux de croissance de la souche ayant une protéase de type $\mathrm{P}_{\mathrm{I}}$, d'autant plus marquée que la proportion de souches ayant une protéase de type $P_{\text {III }}$ dans le mélange est importante (tableau I). Puisque le taux de croissance de la souche ayant une protéase de type $\mathrm{P}_{\mathrm{III}}$ n'est pas affecté, cette interaction peut être qualifiée d'amensalisme [4, 12]. Cet amensalisme est dû aux peptides libérés par la
Figure 1. Pourcentage d'inhibition du taux de croissance en seconde phase exponentielle de quatre souches protéase-positives de Lactococcus lactis cultivées en association avec différents variants protéase-négatifs de $L$. lactis (d'après [11]). Entre parenthèses figure le type de protéase synthétisée par la souche.

$\square, \mathbb{Q}, \mathbb{\nabla}, \square, \mathbf{E}, \mathbf{Z}: \quad$ respectivement L. lactis CNRZ 1075, CNRZ 1259, CNRZ 304, CNRZ 157, CNRZ 429 et $\mathrm{Wg} 2 \mathrm{Prt}^{-}$(souches $\mathrm{Prt}^{-}$).

Figure 1. Percentage of growth rate inhibition of 5 proteinase-positive strains of Lactococcus lactis co-cultured with different proteinase-negative $\left(\mathrm{Prt}^{-}\right)$strains of $L$. lactis (adapted from [11]).

$\square, \bigotimes, \bigotimes, \square, \mathbf{\square}$, 目: L. lactis $\mathrm{Prt}^{-}$ strains CNRZ 1075, CNRZ 1259 , CNRZ 304, CNRZ 157, CNRZ 429 and $\mathrm{Wg} 2 \mathrm{Prt}^{-}$, respectively.

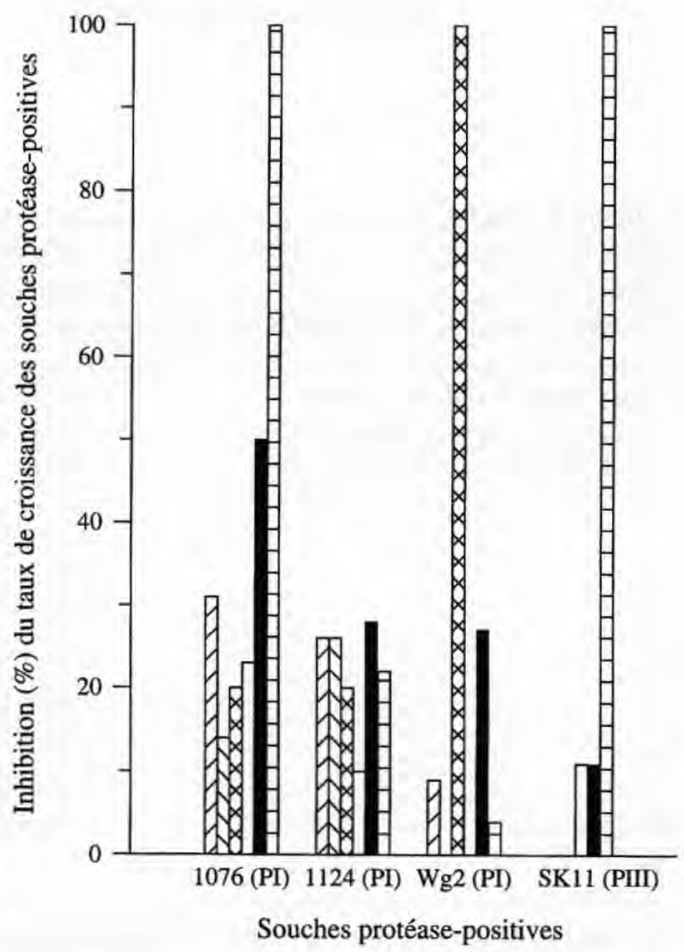




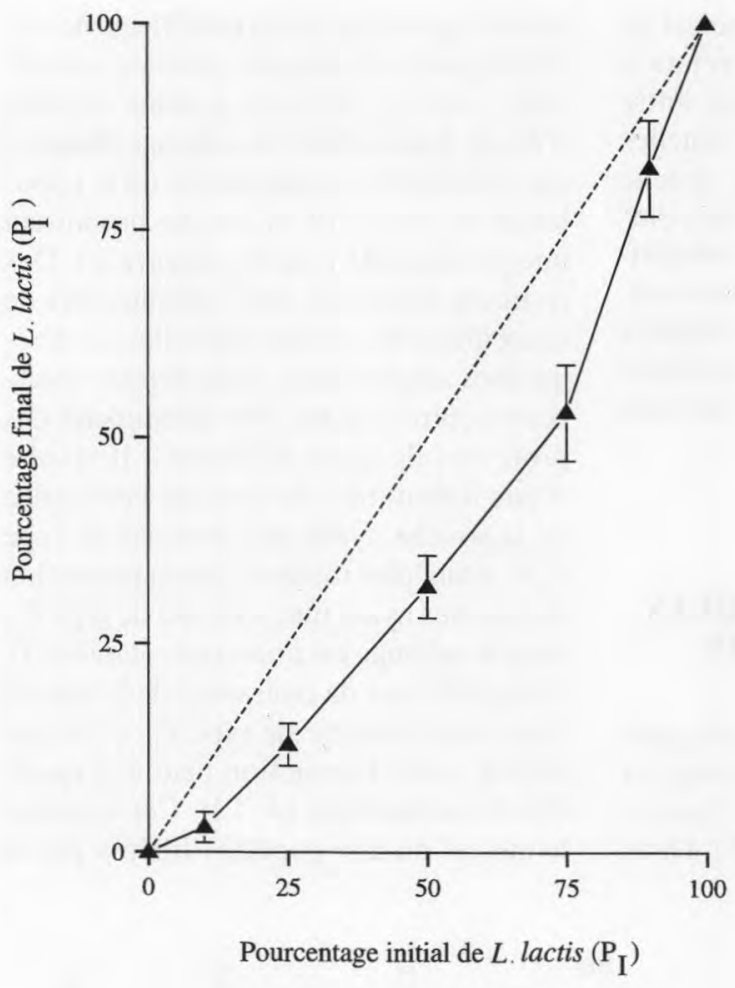

Figure 2. Culture mixte de deux souches de Lactococcus lactis ne différant que par le type de protéase de paroi : évolution de la composition finale du mélange en fonction de sa composition initiale [3]. Entre parenthèses figure le type de protéase synthétisée par la souche.

Figure 2. Changes in population ratio during mixed cultures in milk of two genetically engineered strains of Lactococcus lactis differing only by the type of the proteinase. Between brackets is the type of the proteinase [3].

Tableau I. Taux de croissance de Lactococcus lactis MG611-1 (protéase de type $\mathrm{P}_{\mathrm{I}}$ ) et $L$. lactis SH5-1 (protéase de type $\mathrm{P}_{\mathrm{III}}$ ) cultivés en association dans du lait (d'après [3]).

Table I. Growth rate of Lactococcus lactis MG611-1 ( $\mathrm{P}_{\mathrm{I}}$-type proteinase) and L. lactis SH5-1 ( $\mathrm{P}_{\mathrm{III}}$-type proteinase) co-cultured in milk (adapted from [3]).

\begin{tabular}{lcc}
\hline Pourcentage initial de L. lactis & \multicolumn{2}{c}{ Taux de croissance $\left(\mathrm{h}^{-1}\right)$ de $L$. lactis } \\
\hline SH5-1 $\left(\mathrm{P}_{\mathrm{III}}\right)$ dans le mélange & MG611-1 $\left(\mathrm{P}_{\mathrm{I}}\right)$ & SH5-1 $\left(\mathrm{P}_{\mathrm{III}}\right)$ \\
\hline 0 & $0,74 \pm 0,07$ & - \\
10 & $0,55 \pm 0,07$ & $0,76 \pm 0,08$ \\
25 & $0,58 \pm 0,05$ & $0,78 \pm 0,04$ \\
40 & $0,51 \pm 0,07$ & $0,69 \pm 0,09$ \\
50 & $0,44 \pm 0,03$ & $0,76 \pm 0,08$ \\
60 & $0,42 \pm 0,05$ & $0,76 \pm 0,08$ \\
75 & $0,50 \pm 0,07$ & $0,70 \pm 0,03$ \\
90 & $0,46 \pm 0,04$ & $0,70 \pm 0,08$ \\
100 & - & $0,75 \pm 0,06$
\end{tabular}

Moyennes de quatre répétitions, avec intervalles de confiance à $95 \%$. 
protéase de type $\mathrm{P}_{\mathrm{III}}$. Lorsqu'ils sont ajoutés à une culture pure de la souche ayant une protéase de type $P_{1}$, ils induisent une réduction faible (de l'ordre de $12 \%$ ), mais statistiquement significative $(P=0,05)$ de l'activité protéolytique exprimée par les souches. Néanmoins, cette réduction ne semble pas suffisamment forte pour expliquer l'importance de l'effet inhibiteur. On peut supposer l'existence d'un autre effet des peptides, probablement un effet de compétition entre les peptides libérés par les deux types de protéase pour leur transport intracellulaire.

L'interaction entre souches de lactocoques, qu'elles soient $\mathrm{Prt}^{+}$ou Prt, dépend donc de deux facteurs principaux : le nombre respectif de cellules en présence, et le type de protéase synthétisée par les souches $\mathrm{Prt}^{+}$. Ce rôle prépondérant des protéases de paroi sur l'interaction ne doit pas surprendre, si l'on considère que l'essentiel de la croissance des lactocoques dépend de l'activité protéolytique [10].

\section{INTERACTION ENTRE \\ LACTOCOCCUS LACTIS ET LEUCONOSTOC MESENTEROIDES}

Les effets de l'interaction entre souches de lactocoque et de leuconostoc, au cours de cultures mixtes dans du lait, apparaissent beaucoup plus variables. Certaines études font état d'une inhibition du leuconostoc [13], d'autres au contraire rapportent une stimulation de ce microorganisme [2]. Dans une étude récente [1], 24 cultures mixtes associant une souche de Leuc. mesenteroides et une souche protéolytique de L. lactis ont été analysées.
Figure 3. Évolution du taux de croissance (-) et de la population maximale (A) de Leuconostoc mesenteroüdes cultivé en association avec Lactococcus lactis CNRZ 1076, en fonction de la composition initiale du mélange (d'après [1]).

Figure 3. Changes in growth rate $(\mathbf{)})$ and maximal population $(\boldsymbol{\Delta})$ of Leuconostoc mesenteroides co-cultured with Lactococcus lactis CNRZ 1076 as a function of the initial composition of the mixture (adapted from [1]).
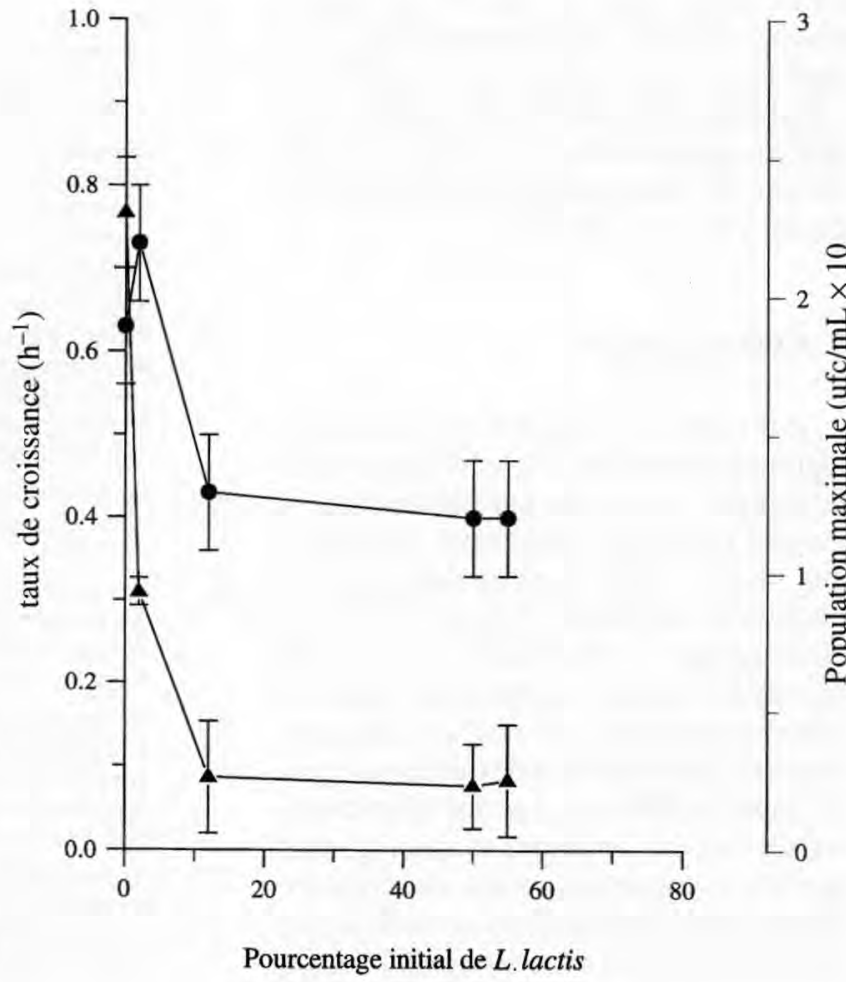

Pourcentage initial de $L$. lactis 
Dans tous les cas, une inhibition partielle de la croissance du leuconostoc était observée, dont l'intensité croissait avec le pourcentage de lactocoques initialement présents dans le mélange (figure 3 ). Des expériences de supplémentation du lait avec divers nutriments ont permis de montrer que l'interaction résultait d'une compétition, entre les deux souches, pour l'utilisation des substrats azotés non protéiques du lait. L'absence d'effet de l'association sur la croissance du lactocoque laisse supposer une bien meilleure efficacité des systèmes de transport des peptides chez ce microorganisme que chez les leuconostocs. Le caractère général des résultats obtenus dans cette étude est à rapprocher de l'observation rapportée par Vedamuthu [14], selon laquelle, au sein des collections de souches de lactocoques et de leuconostocs, la très grande majorité des associations possibles se révèle être néfaste pour le leuconostoc. Cependant, l'existence, même rare, de souches de leuconostocs stimulées par la présence de lactocoques, laisse penser qu'il existe une variabilité importante de la capacité des leuconostocs à utiliser les sources d'azote du lait, ce dont pratiquement rien n'est connu à I'heure actuelle.

\section{CONCLUSION}

Il n'existe pas aujourd'hui de souches regroupant toutes les caractéristiques technologiques souhaitées par l'industrie fromagère (propriété acidifiante, résistance aux phages, activité protéolytique, production de métabolites particuliers). En conséquence, la fermentation industrielle du lait fait généralement intervenir des levains complexes, composés de plusieurs souches appartenant à des espèces et/ou des genres différents, chacune étant caractérisée par des aptitudes technologiques propres. L'existence, au sein de ce levain, de multiples interactions aboutit à une modification de la composition du levain.
De ce fait, les caractéristiques technologiques du levain, prévues d'après le comportement des souches en culture pure, peuvent s'en trouver profondément affectées. La maîtrise de la fermentation lactique requiert donc la connaissance des paramètres régulant la croissance et le métabolisme des souches utilisées, y compris les phénomènes de coopération et/ou d'inhibition qui peuvent se produire entre les souches, Comme l'illustrent les trois exemples présentés, la compréhension des phénomènes d'interaction nécessite une connaissance approfondie de la physiologie des bactéries, en particulier de l'approvisionnement intracellulaire en acides aminés.

\section{RÉFÉRENCES}

[1] Bellengier P., Richard J., Foucaud C., Associative growth of Lactococcus lactis and Leuconostoc mesenteroïdes strains in milk. J. Dairy Sci. 80 (1997) 1520-1527.

[2] Bocquien C.Y., Corrieu G., Desmazeaud M. Effect of fermentation conditions on growth of Streptococcus cremoris AM2 and Leuconostoc lactis CNRZ 1091 in pure and mixed cultures, Appl. Environ. Microbiol. 54 (1988) 2527-2531.

[3] Flambard B., Richard J., Juillard V., Interaction between proteolytic strains of Lactococcus lactis influenced by different types of proteinase during growth in milk, Appl. Environ. Microbiol. 63 (1997) 2131-2135.

[4] Fredrickson A.G., Behavior of mixed cultures of organisms, Annu. Rev. Microbiol. 31 (1977) 63-87.

[5] Harriman L.A., Hammer B.W, Variations in the coagulation and proteolysis of milk by Streptococcus lactis, J. Dairy. Sci. 14 (1931) 40-49.

[6] Helinck S., Richard J., Juillard V., The effects of adding lactococcal proteinase on the growth rate of Lactococcus lactis depend on the type of the enzyme, Appl. Environ. Microbiol. 63 (1997) 2124-2130.

[7] Hugenholtz J., Veldkamp H., Competition between different strains of Streptococcus cremoris, F.E.M.S. Microbiol. Ecol. 31 (1985) 57-62. 
[8] Juillard V., Richard J., Mixed culture in milk of a proteinase-positive and a proteinasenegative variant of Lactococcus lactis subsp. lactis: influence of initial percentage of proteinase-positive cells on the growth parameters of each strain and on the rate of acidification, Lait 74 (1994) 3-12.

[9] Juillard V., Spinnler H.E., Desmazeaud M.J., Bocquien C.Y., Phénomènes de coopération et d'inhibition entre les bactéries lactiques utilisées en industrie laitière, Lait 67 (1987) 149-172.

[10] Juillard V., Foucaud C., Desmazeaud M., Richard J., Utilisation des sources d'azote du lait par Lactococcus lactis, Lait 76 (1996) 13-24.

[11] Juillard V., Furlan S., Foucaud C., Richard J., Mixed cultures of proteinase-positive and proteinase-negative strains of Lactococcus lactis in milk, J. Dairy Sci. 79 (1996) 964-970.

[12] Meers J.L., Growth of bacteria in mixed cultures, Crit. Rev. Microbiol. 2 (1973) 139-184.

[13] Pack M.Y., Vedamuthu E.R., Sandine W.E., Elliker P.R., Leesment H., Effect of temperature on growth and diacetyl production by aroma bacteria in single- and mixed-strain lactic cultures, J. Dairy Sci. 51 (1968) 339-344.

[14] Vedamuthu E.R., The dairy leuconostoc: use in dairy products, J. Dairy Sci. 77 (1994) 2725-2737.

[15] Winkel S.A., Richardson G.H., Cell mass and acid production of proteinase-positive and proteinase-negative lactic cultures in buffered nonfat milk, J. Dairy Sci. 67 (1984) 2856-2859. 